tambour is used for volume changes. The kymograph is the principal recording medium. The experimental methods are not always sufficiently detailed, and some of the methods used are open to criticism. The results on the whole are well presented and summarized; a bibliography in which Russian references predominate is appended to each paper.

The translation is good and the text easily read. The publishers are to be congratulated in presenting this book and the reviewer's only regret is that the original publication was in 1955. A 1960 edition, if such were available, would be even more interesting to read.

\section{Controlled Clinical Trials}

Symposium by the Council for International Organization of Medical Sciences. Pp. vii + 177. Oxford: Blackwell. I960. 20s.

This is a most valuable collection of papers and I would advise without hesitation anyone contemplating carrying out controlled trials to study their contents in detail.

Every contributor brings out some fresh point of special value in his aspect. It is well that Professor Bradford Hill should emphasize that clinical trials are fundamentally for the advancement of knowledge.

Professor L. J. Witts touches a vital factor when he says that the physician should never complicate the position by taking payment for controlled therapeutic trials, though manufacturers should be expected to supply drugs free of charge.

Many interesting controlled trials are described, as, for example, the antihistaminic drugs in the treatment of the common cold, the treatment of sore throats in young adults, treatment of rheumatoid arthritis, pulmonary tuberculosis, coronary thrombosis, certain forms of malignant disease and so on.

In all cases special reference is made to the difficulties encountered. Pulmonary tuberculosis is a disease which lends itself to controlled clinical trials. An adequate number of patients and random allocation of treatment are stressed. The double blind trial is best, for it eliminates the doctor bias.

Dr. Doll aptly describes how in certain diseases, e.g. leukemia, a concurrent assessment of several treatments can be carried out, thereby reducing the time and making the fullest use of the number of patients.

The general principles governing controlled clinical trials are effectively applied to the study of malignant disease.

The would-be worker in clinical trials will find everything he needs in these pages and the book is well worth buying.

\section{Atlas and Manual of Dermatology and Venereology}

Prof. Dr. W. Burckhardt. Translated and edited by Stephan EPstein, M.D. Pp. xii +276 , with 73 illustrations and 99 coloured plates. Baltimore: The Williams \& Wilkins Co. London: Baillière, Tindall \& Cox. 1960. I12s.

To those really interested in dermatology and venereology here is a moderately priced, splendidly illustrated, informative book.

The subject has been adequately covered, apart from the chapter on allergic skin diseases, where the writer leaves the reader with some doubt as to his concept of eczema and dermatitis, especially when he uses such terms as occupational eczema.

Treatment could have been more detailed to advantage, with less emphasis on so many proprietary remedies, some of which are not known as such in this country. Arsenic may be a time-honoured drug, but should have no place in treating lichen planus today.

The advent of griseofulvin just missed inclusion and would have resulted in the rewriting of much of the treatment of dermatomycoses.

The inclusion of references for further reading should be useful to the non-dermatological specialist and others, but I fear the work may prove too comprehensive for the average busy general practitioner, who, for example, has little opportunity to know about the deep mycoses.

\section{Biochemistry}

Abstracts of Sectional Papers and Analytical Index to Symposia and Colloquia of the Fourth International Congress of Biochemistry, Vienna. General Editor: O. Hoffman-Ostenhof. Compiled by the editorial staff of the 'International Abstracts of Biological Sciences'. Pp. 293. Vienna: F. Wewalka. London: Pergamon Press. 1960. roos.

This is the fifteenth volume of the Complete Proceedings of the Fourth International Congress of Biochemistry, which was held in Vienna in 1958. It consists of abstracts of the papers presented at the Congress, and printed in full in the previous volumes, together with complete subject and author indices. Since approximately 2,000 papers were read at the Conference, the abstracts in this volume will help those interested in any aspect of biochemistry to sort out the wheat from the chaff, and to find in the overwhelming mass of material those papers of special interest to themselves. However, at the price of $£_{5}$, few private readers are likely to buy this book, and the editors might have been well advised to bring out a cheaper, paperbacked edition. As a reference source for larger specialized libraries, this is an essential adjunct to the previous 14 volumes.

\section{St. Peter's Hospital for Stone, r860-1960}

Editor: Clifford Morson, o.B.E., F.R.c.s. Pp. viii +64 , illustrated. Edinburgh and London: E. \& S. Livingstone Ltd. 1960. 2 is.

The year 1960 is the centenary of St. Peter's Hospital for the Stone. After some discussion it was decided that the best way to commemorate 100 years of work was to publish a history of the hospital and the people who worked in it. For many years St. Peter's was the only special hospital for the treatment of genito-urinary disease in Great Britain. It was founded much later than the 46 other specialist hospitals already in London at that time. One of the main reasons for establishing yet another specialist hospital was for the better treatment of stones. The incidence of stone was increasing and it was hoped to replace the older methods of cutting for stone by crushing or dissolving them.

In a chapter on the history of the hospital, Cuthbert Dukes tells us how the hospital was moved from Marylebone to Berners Street before being established in Covent Garden, and of the bitter opposition to its foundation by the medical profession and the British Medical Association. (Indeed, some of the earlier surgeons were obliged to resign their appointments because of pressure brought upon them by their colleagues.) He tells us the fascinating story of the anonymous gift to the hospital of $£_{10,000}$ in 1873 and the sequel 45 years later.

Following a short chapter on the management committee, there follows a biography of the past surgeons together with the references of their selected publications, which take up the major part of the book. It would have been of great value if some of these had 